\title{
Numerical investigation of shallow-water effects on hydrokinetic turbine wake recovery
}

\author{
O. El Fajri, S. Bhushan, D. Thompson and T. O'Doherty
}

\begin{abstract}
Thrust, power and intermediate wake predictions obtained using resolved rotating blade with sliding mesh simulations for a hydrokinetic turbine (HKT) are assessed using the open-source flow solver OpenFOAM. Single- and two-phase URANS and DES computations are performed for three-blade, $0.5 \mathrm{~m}$ diameter (D) turbine mounted on a stanchion that intersects the free surface with a tip-speed ratio $\lambda=6.15$. The thrust and power predictions compare within $5 \%$ of the experimental data. Results show that the thrust predictions are dominated by the pressure distribution on the blades, whereas the shear stress plays a significant role in the power predictions. The turbine performance showed unsteadiness with amplitudes around $3 \%$ of the mean, due to the disruption of the flow each time a blade passed in front of the stanchion. The wake recovery is primarily due to the growth of shear layers (originating from the blade tips) towards the turbine axis, which are primarily caused by the cross-plane turbulent velocity. The shear layer growth is enhanced by the turbulence produced by the stanchion. Predictions of the mean wake profile compared within $10 \%$ of the experimental data, which is significant improvement over previous Fluent predictions that showed large errors of $22 \%$. The improved predictions in OpenFOAM is attributed to better turbulence predictions. Two-phase results show that the interaction between the wake and free-surface is initiated by the interaction of stanchion with the freesurface. The free-surface creates a blockage effect that accelerates the flow in the upper bypass region and enhances the wake recovery.
\end{abstract}

Keywords-Hydrokinetic turbine, wake, recovery, free surface.

Manuscript received 16 March; accepted 25 March; published 12 May, 2020. This is an open access article distributed under the terms of the Creative Commons Attribution 4.0 licence (CC BY http://creativecommons.org/licenses/by/4.0/). unrestricted use (including commercial), distribution and reproduction is permitted provided that credit is given to the original author(s) of the work, including a URI or hyperlink to the work, this public license and a copyright notice. This article has been subject to single-blind peer review by a minimum of two reviewers. This effort was partially supported by the Center for Advanced Vehicular Systems,

\section{INTRODUCTION}

$\mathrm{H}$ YDROELECTRIC power represents a clean and renewable source of energy that accounts for $16 \%$ of all electricity generated in the world [1] and is predominately produced by the impoundment of rivers. In contrast, hydrokinetic power, produced by naturally flowing water without impoundment such as river currents, ocean currents, and tidal streams, represents a largely untapped renewable energy source. The primary advantage of the hydrokinetic power is its predictability, as the energy in the tides can be accurately predicted well in advance [2]. While impounded hydropower is a wellunderstood technology, hydrokinetic power generation is an area of active research.

Computational fluid dynamics (CFD) has the ability to assist in design of hydrokinetic turbine farms through assessment of device performance under complex flow conditions [3]. A review of the literature shows that CFD simulations have been used to either understand the effects of free-stream turbulence [4], topography and boundary layer [5, 6], dynamic motions of the platform [7], etc. on power production and loading on turbines; and prediction of turbine wake recovery for a single turbine [8] or those in an array $[9,10]$ to estimate appropriate array design [11]. Some studies have been performed to study the effect of the air-water interface on HKT performance and wake recovery. The following provides a summary of the studies.

Power production and dynamic loading studies have employed high-fidelity, blade-resolved simulations using either Reynolds Averaged Navier Stokes (RANS) or large eddy simulation (LES) turbulence modeling strategies. For example, Afghan et al. [4] performed RANS and LES computations of flow over a three-blade turbine to validate prediction of thrust and power coefficients for a range of tip-speed-ratio $\lambda=4$ to 10 . Results demonstrated that LES

Mississippi State University. The authors also acknowledge the financial support of EPSRC EP/N020782/1 under the Supergen Marine Grand Challenge programme.

O. El Fajri, S. Bhushan, D. Thompson are with Center for Advanced Vehicular Systems, Mississippi State University, Mississippi State, MS, 39762, USA (e-mail: oe72@msstate.edu).

T. O'Doherty is with Cardiff University, Queens Building, The Parade 14-17, Cardiff, CF24 3AA, UK (e-mail: odoherty@cardiff.ac.uk).

Digital Object Identifier https://doi.org/10.36688/imej.3.25-35 
performs better than URANS in predicting the blade tip vortices and their interaction with the supporting tower. Results also demonstrated that wake recovery distance decreases with increasing upstream turbulence. Ahmed et al. [12] performed RANS and LES of flow over HKT to evaluate the fluctuating loads, including thrust, power and bending moment, on turbine blades. They concluded that both URANS and LES predict mean power coefficients in good agreement with experimental data. However, LES predicts a realistic unsteadiness in blade loads, which arise due to flow separation from the blade, blocking effect of the support tower and blade-generated turbulence, and result in up to $\pm 10 \%$ variation over mean loads. Zhang and Kim [13] and Liu et al. [7] performed RANS computations for semi-submersible-type floating offshore wind turbines (FOWTs) under combined wind-wave excitation conditions. Zhang and Kim [13] reported 8 to $10 \%$ increase in the thrust and power predictions for FOWTs compared with an onshore wind turbine due to pitching of the turbine.

Wake prediction studies have been performed using RANS and LES, but using low-fidelity turbine blade models, such as actuator disk or actuator line models. Bouras and Ingham [6] used RANS along with an actuator disk model for prediction of the wake. The results demonstrated that traditional RANS models predict faster wake recovery than experiments and over predict turbulent kinetic energy (TKE). Kasmi et al [14] drew a similar conclusion. Their study reported that the standard $k$ - $\varepsilon$ URANS model, where $k=\frac{1}{2}\left(u^{\prime 2}+v^{\prime 2}+w^{\prime 2}\right)$ is the TKE and $\varepsilon$ is the dissipation, over predicts turbulence kinetic energy in the regions of high mean shear, i.e., in the near/ intermediate wake region behind the blade tip, thereby increasing the turbulent diffusion and under predicting the velocity deficit. They proposed modifications to the turbulence model by adding a dissipation term to limit the turbulent kinetic energy (and viscosity) in the high shear regions. Espinosa [15] performed LES of flow over a wind turbine using actuator disk model. The study reported good predictions of velocity deficit and TKE profiles in the wake (from $x / D=2$ to 10 ), when compared with experimental data. One of the critical modelling aspects emphasized in the study was implementation of resolved turbulence trigger. Wang et al. [10] performed LES of flow over three yawed wind turbines arranged in different wake configurations using actuator-line model. The CFD predictions compared very well with experimental data for wake behaviour and power output. Archer et al. [16] used LES and an actuator-line model to quantify the effects of array layout on the performance of offshore wind farms. Moon et al. [17] used the above numerical approach to generate wake profiles of wind turbines. The wake-related mean and turbulence field were then estimated to develop a stochastic wake model for farm design.

Bahaj et al. [18] investigated the effect of topography and the free-surface on turbine performance using a porous disc model. The study reported that the closeness of the free surface to the turbine induces axial asymmetry in the wake. Riglin et al. [19] performed numerical analysis of hydrokinetic turbine power production using a resolved blade model with four different tip clearances (i.e., gap between the blade tip and the free-surface) ranging from $C$ $=0.57 \mathrm{D}-2.28 \mathrm{D}$, where $\mathrm{D}$ is turbine diameter. The tip clearances corresponded to critical Froude number $\operatorname{Frc}_{\mathrm{C}}=$ 1.31 to 0.71 . Results showed a $32.2 \%$ reduction in turbine power at critical flow conditions, i.e., $\operatorname{Frc}_{2} \geq 1$, and at these conditions the turbine wake started to interact with the free surface at around 4 to 5D downstream of the turbine and shift upwards. Kolekar et al. [20] investigated the effect of channel blockage and tip clearances on threeblade marine HKT power production both experimentally and using blade resolved simulations. The tip clearances were varied from $C=0.03 \mathrm{D}$ to $0.73 \mathrm{D}$. They concluded that channel blockage improves the power productions, whereas the free-surface interaction reduces the power production when tip clearances are less than 0.25D. Smaller tip clearances, i.e., $C \leq 0.2 \mathrm{D}$, also result in a 5 to 10 $\%$ drop in water depth behind the turbine, which significantly affects the wake expansion and recovery.

Overall, the literature review shows that fullyresolved blade model has been used primarily for studies to evaluate loading on turbine blade, whereas wake studies have primarily employed on the actuator disk/line models with high fidelity LES to either optimize array layout or to develop wake models to be used for array design [21].

The objective of this research is to validate the predictive capability of blade-resolved simulations for HKT thrust, power and intermediate wake predictions using flume data [2]. The study builds on the authors' previous blade-resolved HKT validation effort using Ansys/Fluent using RANS, Detached eddy simulation (DES) and LES models [22,29]. Their study demonstrated that, although blade-resolved simulations are sufficiently accurate for HKT thrust and power predictions, they exhibit deficiencies in the prediction of wake recovery. Typically, the recovery was significantly under predicted compared to experimental data. The LES simulations were somewhat better than URANS, but both failed to accurately predict the TKE levels in the tip vortex region. In the LES simulations, the limitations were clearly caused by issues at the rotating domain interface, as resolved turbulence generated at the blade tips was not transported across the interface. It was difficult to determine whether this limitation was due to grid design, i.e., use of tetrahedral meshes (even though the grid was adaptively refined in the tip vortex region) or solution interpolation at the rotating interface. In this study, OpenFOAM simulations are performed using RANS and DES and two different grid topologies, i.e., mixed hexahedral/tetrahedral cells, and hexahedral cells using SnappyHexMesh a mesh generator supplied with OpenFOAM. Simulations are also performed for twophase flows to accurately depict the experimental 
conditions. The predictions are compared with experimental data and with Fluent results to assess the current state-of-art in predictive capability of bladeresolved simulations.

\section{NUMERICAL METHODS}

Simulations are performed using OpenFOAM, an opensource finite volume CFD toolbox developed by Jasak et al. [23]. The flow fields are governed by the two-phase incompressible Navier-Stokes equations for which the mass and momentum conservation equations are:

$$
\begin{gathered}
\nabla \cdot \rho \boldsymbol{u}^{r}=0 \\
\frac{\partial \rho \boldsymbol{u}}{\partial t}+\nabla \cdot\left(\rho \mathbf{u}^{r} \mathbf{u}\right)+\boldsymbol{\omega} \times \boldsymbol{u} \\
=-\nabla \mathrm{p}+\nabla \cdot\left\{2\left(\mu+v_{T}\right) \boldsymbol{D}\right\}+\rho \boldsymbol{f}_{\boldsymbol{b}}
\end{gathered}
$$

where, $\boldsymbol{u}$ is a velocity vector, $\mathbf{u}^{\boldsymbol{r}}=\boldsymbol{u}-\boldsymbol{\omega} \times \boldsymbol{r}$ is the relative velocity, $\boldsymbol{\omega}=\left(\omega_{x}, 0,0\right)$ is the turbine rotation vector, $\boldsymbol{r}$ is the radial location vector, $\boldsymbol{D}$ is the rate-of-strain tensor, $\boldsymbol{f}_{\boldsymbol{b}}$ is the body force term due to gravity and surface tension, $\mu$ is the molecular dynamic viscosity, $v_{T}$ is the turbulent dynamic viscosity, $\rho$ is the density of the fluid, and $p$ is the kinematic pressure. For single-phase simulations, $\rho$ and $\mu$ are constant and specified to be those of water. For twophase simulations, an addition transport equation is solved for volume-of-fluid (VOF) which tracks the air and water phases of fluid separately, i.e.,

$$
\frac{\partial \alpha}{\partial t}+\nabla \cdot(\boldsymbol{u} \alpha)=0
$$

$\alpha$ is the phase fraction whose value varies between $0 \leq \alpha \leq$ 1. Note that $\alpha=0$ represents the air and $\alpha=1$ represents the water and in between is the air-water interface. The density and viscosity of the mixture are defined as:

$$
\begin{gathered}
\rho=\alpha \rho_{l}+(1-\alpha) \rho_{g} \\
\mu=\alpha \mu_{l}+(1-\alpha) \mu_{g}
\end{gathered}
$$

where the $g$ and $l$ subscripts denote the air and water phases, respectively. For the two-phase simulations performed herein, surface tension effects were neglected, and the body force term (i.e., gravity) is applied only to the water phase.

In a previous study, Robertson et al. [24] validated OpenFOAM numerical methods and turbulence models for incompressible, bluff-body, single-phase flows, which included flow over a backward facing step, a sphere and a sharp leading-edge delta wing. The study identified the $2^{\text {nd }}$-order linear upwind and blended $1^{\text {st }} / 2^{\text {nd }}-$ order bounded central difference schemes as the most efficient for RANS and DES simulations, respectively. These numerical guidelines are adopted in this study.

Simulations were run on 60 processors for about $318 \mathrm{~K}$ time steps, which corresponds to 111 turbine blade rotations, and each time step took 11.1s of CPU time. The total CPU run time was 40 days and 58.3K CPU hours.

\section{SURVEY OF EXPERIMENTAL DATA}

Model-scale experiments focusing on measurements of turbine performance, i.e., thrust $\left(C_{T}\right)$ and power $\left(C_{P}\right)$ coefficient, and intermediate wake $(x / D=1.5-7$, where $x$ is the streamwise direction) characteristics were performed in a recirculating flume at the University of Liverpool $[2,25,26]$. The model HKT had a blade diameter $D=0.5 \mathrm{~m}$ and could have up to six blades [25]. The experiments were performed using a three-blade model (Fig. 1) for different pitch angles. The blade has a Wortmann FX 63-137 profile with a chord length of $0.03 \mathrm{~m}$ at the tip and $0.08 \mathrm{~m}$ at the root with a $35^{\circ}$ twist along the length. The turbine was supported from above using a cylindrical stanchion located $0.3 \mathrm{~m}$ (or $0.6 \mathrm{D}$ ) behind the blades and the turbine axis was at a water depth of $0.425 \mathrm{~m}$. Thus, in the transverse (y) direction, the tip of the turbine was $0.175 \mathrm{~m}$ (or $0.35 \mathrm{D}$ ) below the free-surface, and $0.125 \mathrm{~m}$ (or $0.25 D$ ) above the bottom wall. The test section had a spanwise $(z)$ extent of $1.35 \mathrm{~m}$, thus the blade tip clearance was $0.425 \mathrm{~m}$ (or $0.85 D$ ).

Mason-Jones et al. [24] performed experiments using blade pitch angles of $3^{\circ}, 6^{\circ}$ and $9^{\circ}$, and an incoming water velocity of $U_{0}=1 \mathrm{~m} / \mathrm{s}$. The thrust, power and torque were measured for different blade rotation velocities from $\omega_{0}=11$ $\mathrm{rad} / \mathrm{s}\left(\lambda=\omega_{0} D / 2 U_{0}=2.7\right)$ to $\omega_{0}=28 \mathrm{rad} / \mathrm{s}(\lambda=7)$. Morris [25] identified that the above data had a high level of scatter as: (1) there was an insufficient distance between the turbine rotation plane and the support stanchion; and (2) the coupling between the turbine and the motor coupling was located above the water surface. The experimental set-up was modified, and measurements were repeated for a blade pitch angle of $6^{\circ}$ and $U_{0}=1 \mathrm{~m} / \mathrm{s}$. The thrust, power and torque were measured for $\omega_{0}=18 \mathrm{rad} / \mathrm{s}(\lambda=4.4)$ to $\omega_{0}=28$ $\mathrm{rad} / \mathrm{s}(\lambda=7)$. As shown in Figure 2(a), $C_{P}$ measurements from experiments [24,25] agreed well for $\lambda=4.4-5$. However, some of the data [23] showed higher $C_{P}$ values for $\lambda>5$ and a $5 \%$ higher $C_{T}$ (Fig. 2b).

Tedds et al. [2] performed experiments using a blade pitch angle of $6^{\circ}$ for $\lambda=6.15$, and an inflow velocity range of $U_{0}=0.5-1.5 \mathrm{~m} / \mathrm{s}$ with a turbulence intensity $(T I)$ of $2 \%$. Wake measurements were performed for $x / D=1.5$ to 7 that included: contours of streamwise, transverse and spanwise velocities in five transverse planes at $y / D=0$, \pm 0.25 and $\pm 0.5 ; u^{\prime}$ and TKE profiles with respect to $z / D$ in the plane at $y / D=0$; maximum Reynolds stresses and TKE in the plane at $y / D=0, \pm 0.25$ and \pm 0.5 planes; and a turbulence anisotropy map in the wake. 


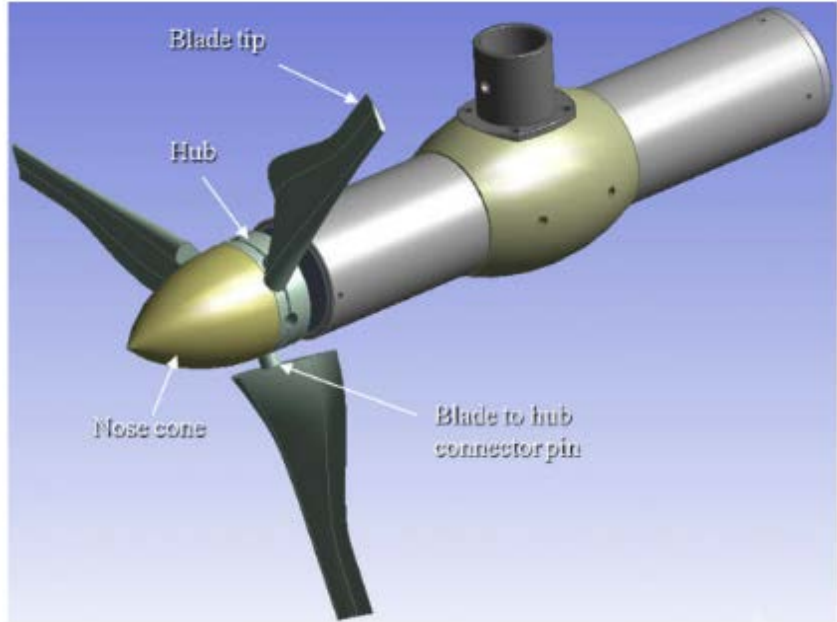

Figure 1: Photograph of the turbine blade and support assembly (taken from [2]).

\section{DOMAIN, GRID AND SIMULATION CONDITIONS}

Simulations were performed for the three-blade HKT with blade pitch angle of $6^{\circ}$ as used in the experiments [2,25]. Simulations are performed for $D=0.5 \mathrm{~m}, U_{0}=0.892 \mathrm{~m} / \mathrm{s}$, inflow turbulence intensity $T I=2 \%$, blade rotation $\omega_{0}=21.932 \mathrm{rad} / \mathrm{s}, \lambda=6.15$ and diameter based Reynolds number $R e_{D}=4.5 \times 10^{5}$ (assuming water at $T=20^{\circ} \mathrm{C}$ ). The flow conditions corresponded to the experimental conditions reported in [2].

As shown in Fig. 3, the simulation domain consisted of two blocks - a hexahedron outer block, and a cylindrical blade assembly block with the turbine center located at the origin $(x=0, y=0$ and $z=0)$. The outer domain extended from $x / D=[-5,21], y / D=[-0.75,1.15]$ and $z / D=[-1.35,1.35]$. The $y$ and $z$ extents of the domain were same as that of the dimensions of the test section, i.e., the cross-section of the flume below the free-surface. The blade assembly cylindrical domain had a diameter of $1.4 D$ and spanned from $x / D=[-0.22,0.38]$.

A Dirichlet boundary condition was used at the inlet. A steady inflow velocity $U_{0}$ was specified and the modeled turbulence parameter was adjusted to produce the appropriate TI. A fixed pressure boundary condition was used at the outlet. A wall-function boundary condition was used both for the blade, stanchion surfaces, and bottom and side boundaries. A sliding interface was used to transfer information between the rotating turbine assembly domain and the static outer domain. The flow in the outer domain was solved in an Earth-fixed inertial reference frame, i.e., $\omega_{x}=0$ in Eq. 1(b). The flow in the turbine assembly domain was solved in an Earth-fixed inertial reference frame with a physical rotation of $\omega_{x}=\omega_{0}$.

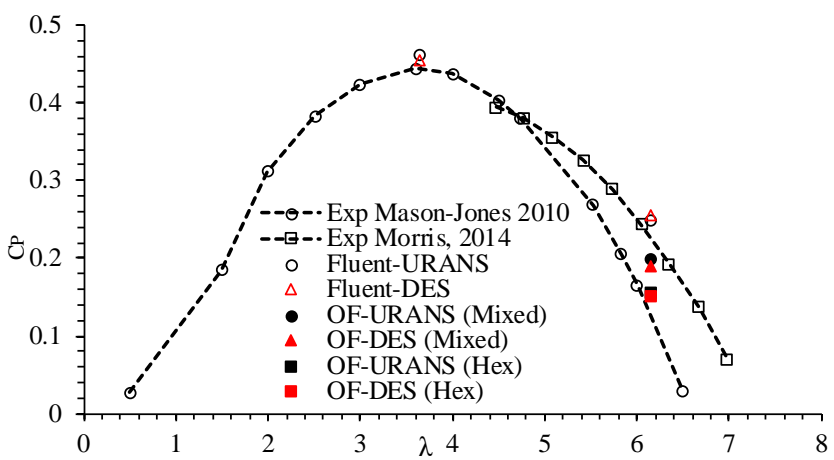

(a)

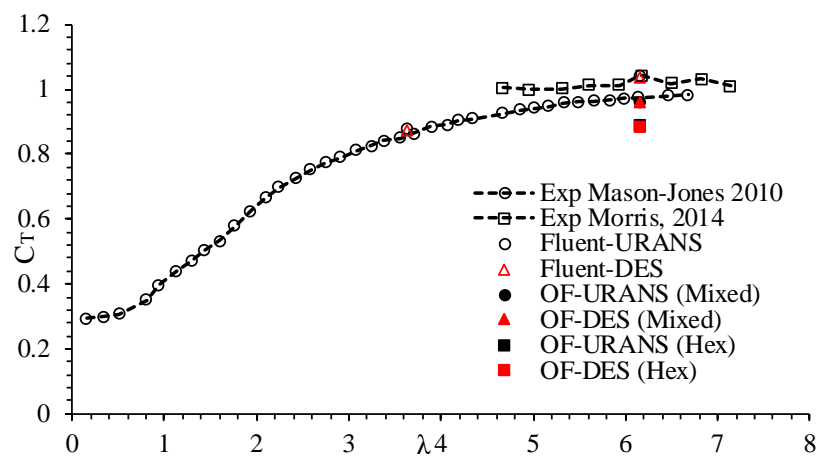

(b)

Figure 2: (a) Power coefficient $C_{P}$ and (b) thrust coefficient $C_{T}$ predicted by URANS and DES for different $\lambda$ are compared with experimental data.

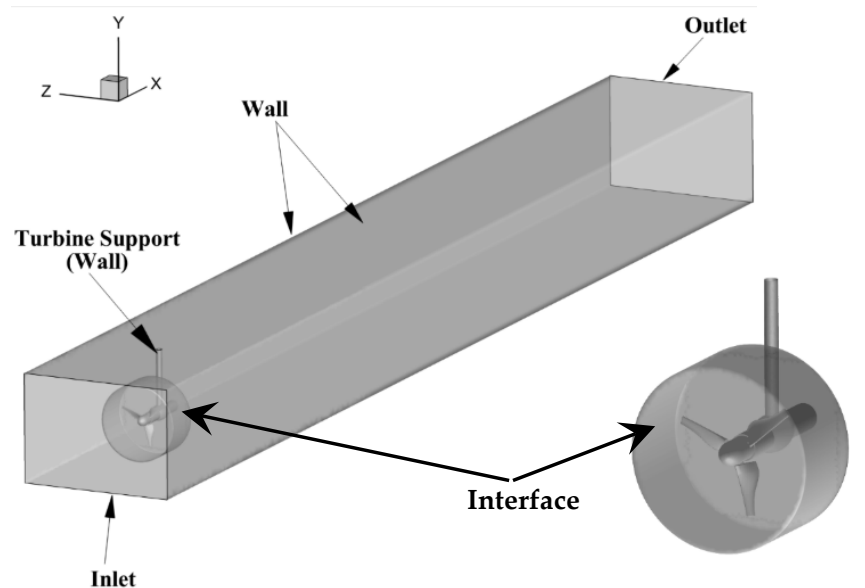

Figure 3: Simulation domain and boundary conditions. Inset figure shows the rotating cylindrical domain for turbine blades and hub. 


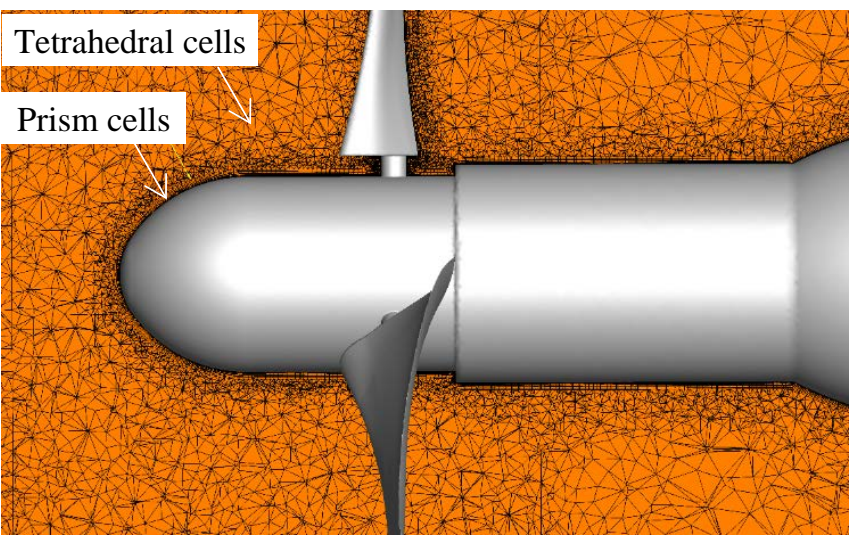

(a)

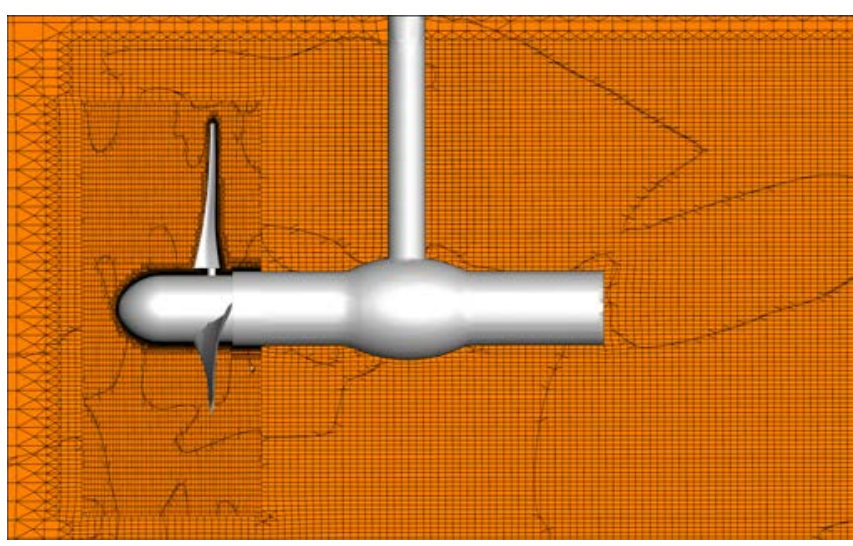

(b)

Figure 4: (a) Mixed prism and tetrahedral cells mesh; and (b) hex cells mesh used in this study.

Table 1: Summary of simulations performed in the study.

\begin{tabular}{|c|c|c|c|c|c|c|c|c|c|}
\hline \multirow{2}{*}{ Solver } & \multirow{2}{*}{ Grid } & \multirow{2}{*}{ Model } & \multirow{2}{*}{ Phase } & \multicolumn{3}{|c|}{ Thrust } & \multicolumn{3}{|c|}{ Power } \\
\hline & & & & $C_{T}(E \%)$ & $C_{T, p}\left(\% C_{T}\right)$ & $C_{T, f}\left(\% C_{T}\right)$ & $C_{P}(E \%)$ & $C_{P, p}\left(\% C_{P}\right)$ & $C_{P, f}\left(\% C_{P}\right)$ \\
\hline \multicolumn{3}{|c|}{ Experiment (Avg.) } & Two & 1.01 & - & - & 0.2 & - & - \\
\hline \multirow{2}{*}{ Fluent [19] } & $8.8 \mathrm{M}$ & URANS & \multirow{2}{*}{ Single } & $1.042(3 \%)$ & $1.034(99.2 \%)$ & $0.013(1.2 \%)$ & $0.248(19.3 \%)$ & $0.342(137.9 \%)$ & $-0.094(-37.9 \%)$ \\
\hline & Mixed cells & DES & & $1.036(2.5 \%)$ & $1.022(98.6 \%)$ & $0.014(1.3 \%)$ & $0.255(21.5 \%)$ & $0.357(140 \%)$ & $-0.102(-40 \%)$ \\
\hline \multirow{5}{*}{$\begin{array}{c}\text { OpenFOAM } \\
\text { (this study) }\end{array}$} & $11 \mathrm{M}$ & URANS & \multirow{4}{*}{ Single } & $0.968(-4.4 \%)$ & $0.96(99.2 \%)$ & $0.006(0.7 \%)$ & $0.198(-1 \%)$ & $0.303(152.8 \%)$ & $-0.105(-53 \%)$ \\
\hline & Mixed cells & DES & & $0.959(-5.3 \%)$ & $0.952(99.2 \%)$ & $0.008(0.7 \%)$ & $0.191(-5.2 \%)$ & $0.294(154.3 \%)$ & $-0.104(-54.7 \%)$ \\
\hline & $7 \mathrm{M}$ & URANS & & $0.890(-13.4 \%)$ & $0.879(98.7 \%)$ & $0.0107(1.2 \%)$ & $0.155(-29 \%)$ & $0.291(187.2 \%)$ & $-0.135(-87 \%)$ \\
\hline & Hex cells & DES & & $0.886(-13.9 \%)$ & $0.875(98.7 \%)$ & $0.0106(1.1 \%)$ & $0.152(-31.5 \%)$ & $0.287(188.1 \%)$ & $-0.135(-88.8 \%)$ \\
\hline & $\begin{array}{c}19 \mathrm{M}, \\
\text { Hex cells }\end{array}$ & URANS & Two & $0.901(-10.8 \%)$ & $0.890(98.8 \%)$ & $0.09(1.2 \%)$ & $0.220(10 \%)$ & $0.322(150.9 \%)$ & $-0.112(-50.9 \%)$ \\
\hline
\end{tabular}

Two different grid topologies were used for the simulations: (1) mixed prismatic- and hexahedral-cell mesh; and (2) hexahedral-cell mesh. The mixed cells mesh was manually generated using Pointwise software [28]. For this grid, first a triangular surface mesh was generated on the boundaries. The boundary cells were then extruded normal to the surfaces by specifying extrusion length to obtain prismatic cells in the boundary layer. The extrusion length was estimated using $R e_{D}$ and analytical turbulent flat plate boundary layer profiles to maintain $y^{+} \sim 1$ for the first cell away from the wall. To capture the boundary layer, around 10 layers were extruded from the surface using an expansion ratio of 1.1. The hexahedral meshes were created using OpenFOAM mesh utility SnappyHexMesh [23], a 3D Cartesian-based mesh generation tool. For SnappyHexMesh, the body geometry file and background hex-cell mesh which corresponds to the computational domain were provided to the mesh generator, they undergo cell splitting and surface snapping. The mesh refinement in the regions of interest was obtained by adding refinement blocks. Close-up view of the grid designs are shown in Fig. 4(a,b).

As summarized in Table 1, single-phase URANS and DES computations were performed using $11 \mathrm{M}$ mixed-cell and 7M hex-cell grids, and two-phase URANS simulations were performed using a $19 \mathrm{M}$ hex-cell grid. Note that the two-phase simulation grid was much larger than the single-phase simulations grid, as an additional refinement block was added near the free surface region.

\section{RESULTS AND DISCUSSION}

\section{A. Performance Predictions}

The $2^{\text {nd }}$-order, bounded-central difference scheme provided more accurate power predictions than $2^{\text {nd }}$-order upwind scheme because of improved wall shear stress predictions on the on the leeward side of the blade (results not shown). Simulations on hex cells under predicted thrust and power compared to predictions mixed cells, because of lower surface pressures and mostly over predicted of blade shear stress towards the tip. As shown in Figure 2 and Table 1, $\mathrm{C}_{\mathrm{T}}$ and $\mathrm{C}_{\mathrm{P}}$ predictions obtained using the $2^{\text {nd }}$-order bounded-central difference schemes on mixed cells compared within $4 \%$ of the experimental data. The poor predictions on the hex-cells were probably because the blade boundary layer was not predicted well by the cut-cell grids. Both thrust and power were primarily due to the pressure and stress distributions over the blades, and hub contributions were $<2 \%$. The thrust was primarily due to pressure forces, whereas both stress and pressure components were important for power predictions. The viscous moments were around $35 \%$ of the pressure moments and acted in the opposite direction of the latter. The Fluent simulations showed similar contributions of pressure and stress moments.

Analysis of the pressure distribution on the blade showed high pressure on the front face and low pressure on the back face, near the blade tips. The pressure difference between the front and back faces was the primary source of drag. For the power predictions, the high and low pressures on the front and back faces, respectively, acted opposite to each other. Whereas the 
high shear stresses towards the blade tips on both the front and back faces resulted in power loss. Thus, it is reasonable that shear stresses played a larger role in power predictions than for thrust.

$C_{T}$ and $C_{P}$ time histories in Fig. 6 showed unsteady variations with amplitudes around $3 \%$ relative to the mean. The variations showed a dominant frequency $f=10$ $\mathrm{Hz}$, which was close to $N_{B} \times \omega_{0} / 2 \pi$, where $N_{B}$ is the number of blades. A similar dominant unsteadiness was not predicted in simulations without the stanchion (results not shown); thus, the unsteady thrust and power predictions were attributed to the disruption of the flow each time a blade passes in front of the stanchion. Such blade-tower interaction is well documented in the literature [4,24].

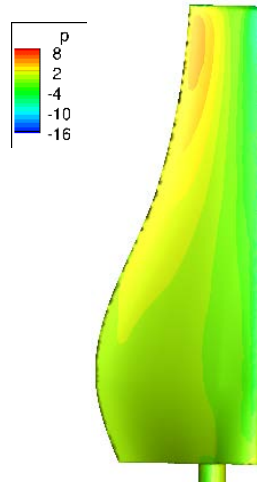

(a)

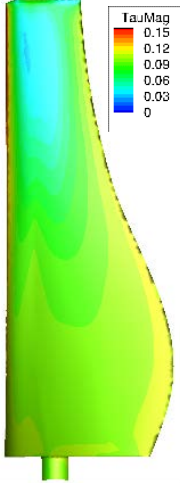

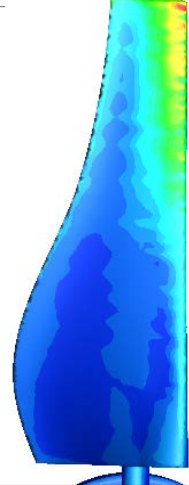

(b)

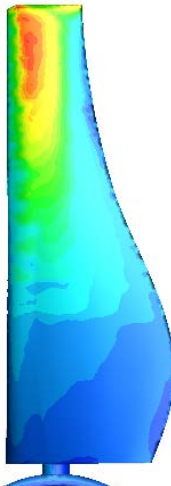

Figure 5: Blade surface (a) pressure and (b) shear stress magnitude distribution on front face (left) and back face (right) obtained using

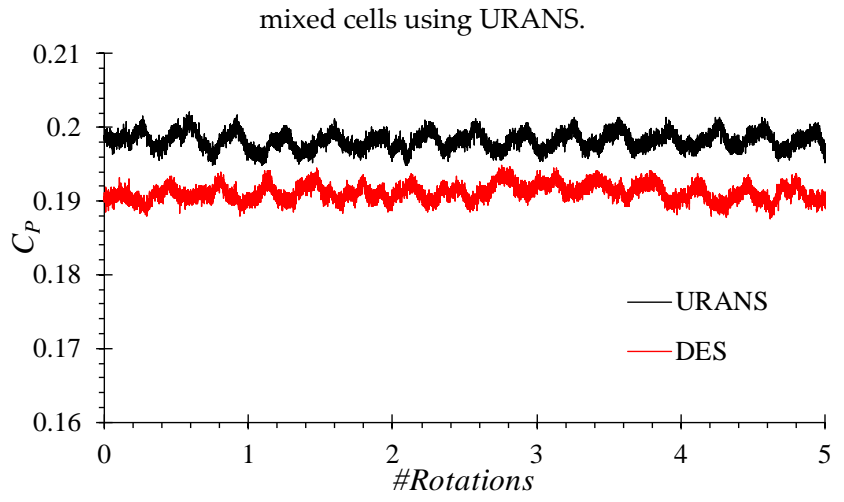

Figure 6: Time history of $C_{P}$ predictions obtained using URANS and DES computations.

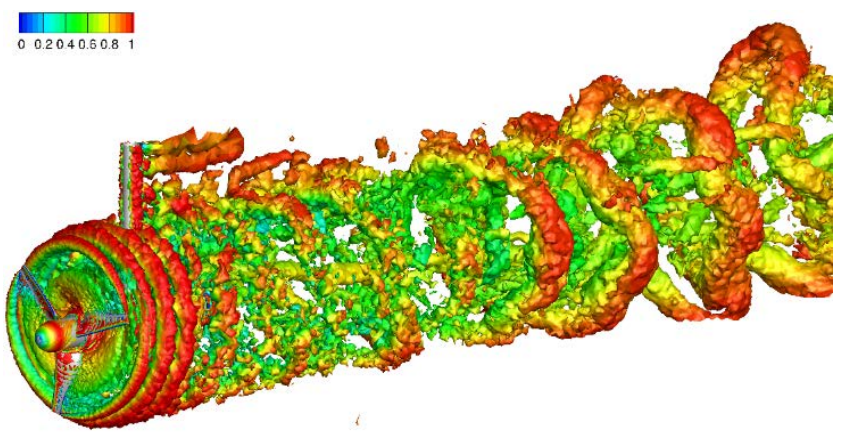

(a)

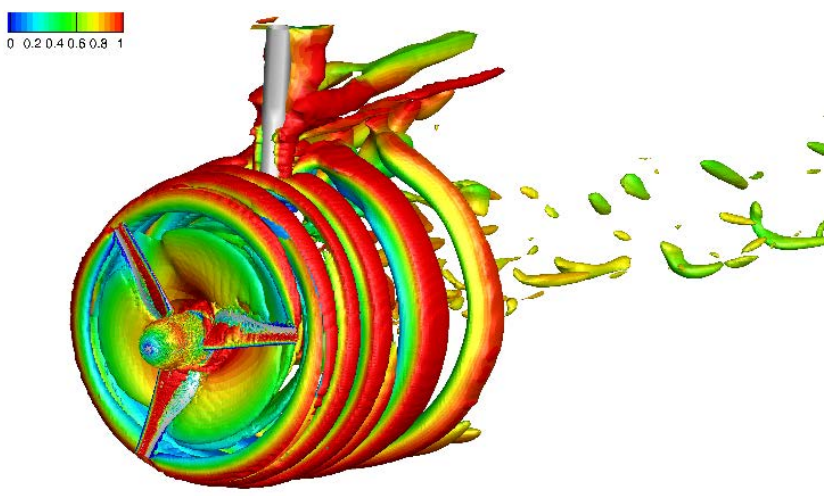

(b)

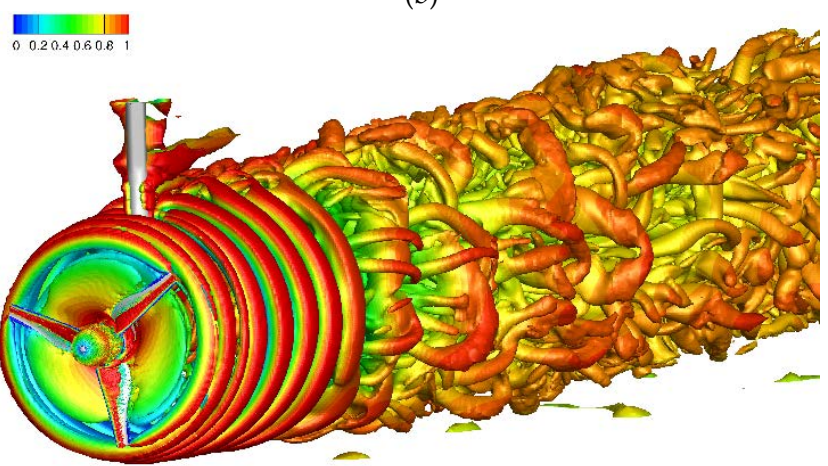

(c)

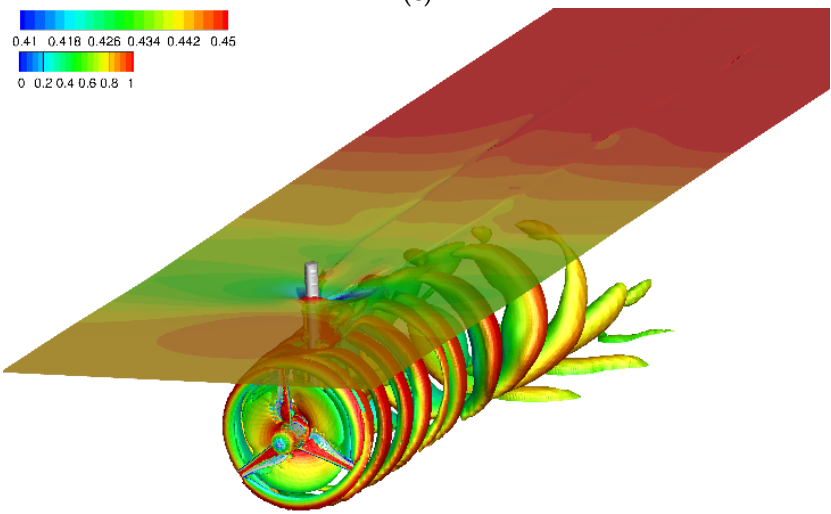

(d)

Figure 7: Instantaneous wake is shown using isosurface of second invariant of rate-of-strain tensor $Q=5$ coloured using streamwise velocity. (a) DES predictions on mixed prism/tetrahedral cells mesh; (b) URANS and (c) DES predictions on hex cells mesh (d) URANS two phase flow along with isosurfaces of the free surface coloured using wave elevation height.

\section{B. Wake Recovery}

Instantaneous wake flow in Fig. 7 showed that the wake region was dominated by large-scale spiralling vortical structures generated from the tip of the turbine blades. The tip vortices expanded slowly outwards and resulted in an annular inverted jet with two shear layers, one developing inward towards the turbine axis and other appearing outward of the blade tip region. The inner shear layer growth was significantly affected by the turbulence generated by the turbine, and thus played a critical role in wake recovery. Predictions also showed spiral vortex rings emerging from the root of the blades. The root vortices showed strong interaction with the turbine-shaft boundary layer and the flow separation behind the shaft. The root vortex ring shrunk in diameter and dissipated much quicker than the tip vortices. 
URANS predicted steady vortical structures which decay rapidly by 2-3D downstream whereas in DES the ring vortices were observed even in far wake up to 10-15D. The vortex ring disintegrated once it interacted with the stanchion, and coherent turbulent structures were generated. As evident, the coherent structures were predicted significantly better on the hex-cell mesh than on tetrahedral cell mesh. The free-surface prediction in the two-phase simulation shows a standing wave pattern with higher wave elevation upstream of the stanchion and lower wave elevation in the wake (Fig. $7 \mathrm{~d}$ ). The freesurface also shows a prominent scar pattern, which is sustained up to 10D downstream in the wake. This scar pattern could be due to the effect of tip vortices. Similar scar patterns have been reported in ship flows due to vortices generated from the hull in the water [30].

The wake deficit profile in the far-wake, beyond, i.e., $x / D=8$, showed a self-similar Gaussian profile. The wake deficit amplitude and the width show a linear variation with $x / D$. The wake amplitude decayed at a rate of $0.185 U_{0} / D$, whereas the wake width increased. The wake width increase was consistent with those reported by Bastankhan and Porte-Agel [27].

\section{Mean Wake Predictions}

The experimental data in the plane at $y / D=0$ in Fig. 8(a) showed a peak mean wake deficit $\left(1-u / U_{0}\right)$ of $71 \% U_{0}$ at $z / D \sim \pm 0.47$, which is directly downstream of the blade tips.

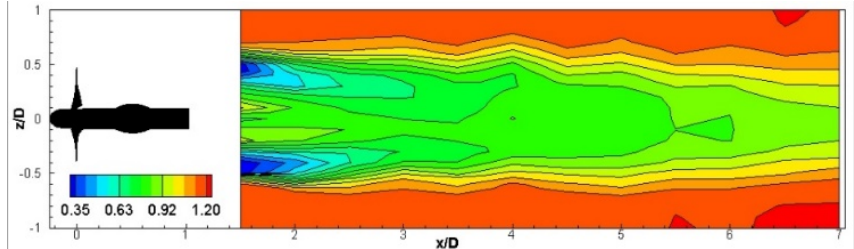

(a)

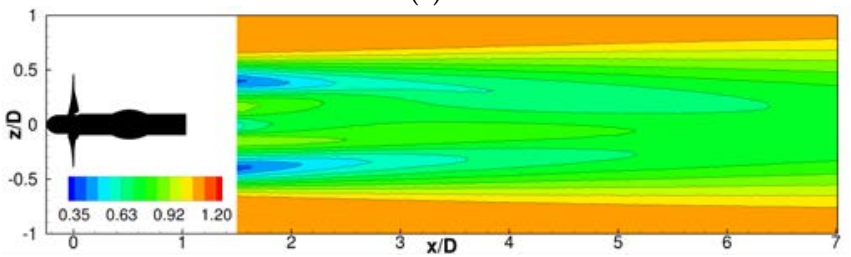

(b)

Figure 8: Mean streamwise velocity contour: (a) experiments and (b) OpenFOAM on mixed cells mesh.

The deficit peak moved towards the axis and reached the centerline by $x / D=5.5-6$, where the deficit is about $16 \% U_{0}$. The deficit contours in the planes at $y / D= \pm 0.25$ were qualitatively similar to those in the plane at $y / D=0$ except that the peak occurred at $z / D \sim \pm 0.32$, slightly inward of the tip location on the plane at $z / D \sim \pm 0.43$. The mean deficit behind the blade tips in the planes at $y / D= \pm 0.5$ showed a peak value around $z / D \sim 0$, which decreased gradually in the downstream direction. The stanchion side planes at $y / D=0.25$ and 0.5 showed $2 \%$ and $6 \%$ higher deficits throughout the intermediate wake, respectively, compared to those in the center plane at $y / D=0$. On the other hand, the deficit in non-stanchion side planes at $y / D=-0.25$ and -0.5 showed $5 \%$ and $14 \%$ lower wake deficits, respectively.

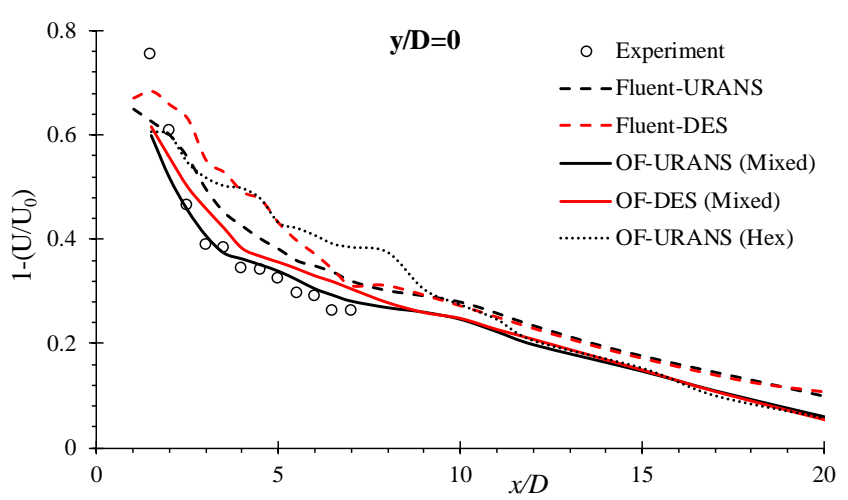

(a)

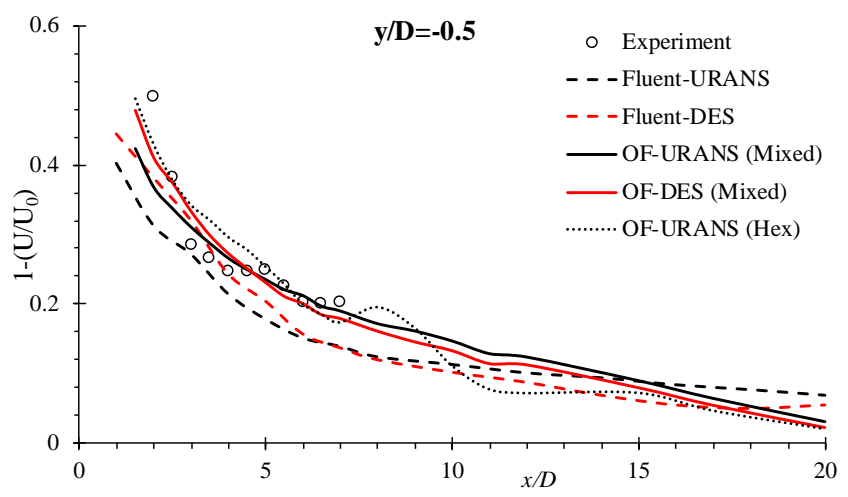

(b)

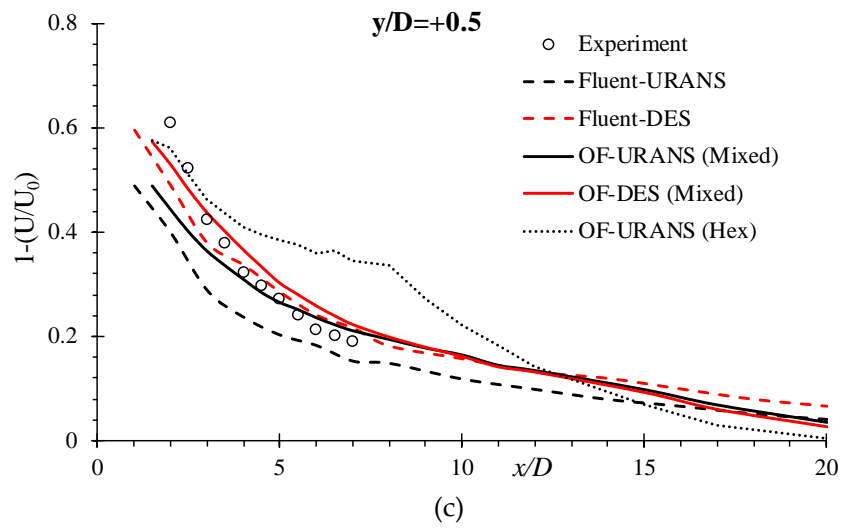

Figure 9: Validation URANS and DES wake predictions using experiment [2]. Variation of peak wake deficit downstream of turbine at: (a) $y / D=0$ and (b) $y / D=-0.5$ and (c) $y / D=0.5$ planes.

The CFD predictions were qualitatively consistent with the experimental data, as shown in Figs. 8(b). The CFD predictions also showed higher wake deficit towards the stanchion side than on a deep-water side similar to the experiments. To validate the predictions, the peak wake deficits in the planes at $y / D=0, \pm 0.25$ and \pm 0.5 were compared with the experimental data. Sample results in the planes at $y / D=-0.5,0$ and 0.5 are shown in Fig. 9. Both URANS and DES predictions compared quite well with the experimental data, and the averaged error is $8.2 \%$, $10.1 \%$ and $12.2 \%$ for $y / D=-0.5,0$ and 0.5 , respectively. The OpenFOAM results were significantly better than those predicted using Fluent, where the latter showed an average error of $22 \%$. The wake recovery was also 
predicted better in OpenFOAM than Fluent, although the shear-layer reached the centerplane further downstream than the experiment.

The experimental data in the $y / D=0$ plane in Fig. 10(a) showed negative and positive mean transverse velocities (v) for positive and negative $z / D$, respectively. The peak $|v| \sim 0.12 U_{0}$ was observed at $x / D \sim 1.5$ and decreased downstream. The peak location showed asymmetry and the profile tended to shift towards the negative $z / D$ direction. CFD predictions showed negative and positive $v$ contours consistent with the experiment. Analysis of the instantaneous solution showed that the antisymmetric velocity pattern was predicted due to the counterclockwise swirl imparted by clockwise rotating turbine in the wake. The peak $|v|$ was obtained around $z / D \sim 0.3$, which is in between the blade tip and the root. The CFD predictions also showed a more diffused profile that the experiment.

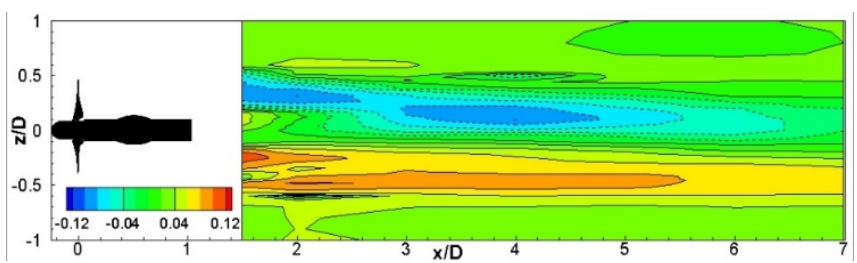

(a)

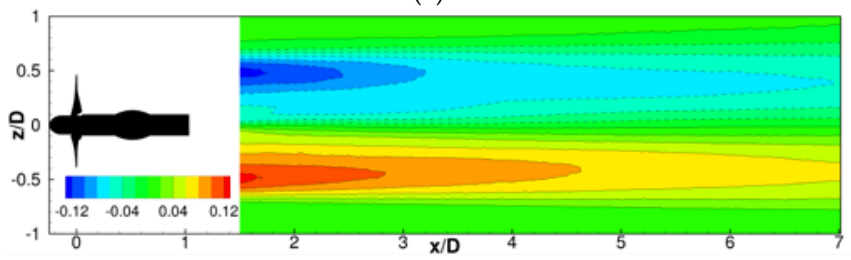

(b)

Figure 10: Mean transverse velocity contour: (a) experiments and (b) OpenFOAM on mixed cells mesh.

The experimental data showed mostly small mean spanwise velocities with peak values $|w|<0.05 U_{0}$ (figure not shown). The spanwise velocity didn't not show any well-defined structure on other planes. The CFD predictions also showed almost negligible spanwise velocity with $|w|<0.1 U_{0}$.

\section{Turbulent Wake Predictions}

The TKE profiles in the intermediate wake predicted by the simulations in the plane at $y / D=0$ were compared with the experiment data in Figure $11^{1}$. The experimental data showed large TKE values close to the blade tips around $z / D=0.55$ for $x / D \leq 2$. The TKE peak moved slightly towards the axis, and an almost uniform TKE was observed at $x / D=4.5$. The peak TKE value decreased with $x / D$, where the rate of decay is much higher close to the turbine. The data also showed high TKE towards the axis at $x / D=1.5$, which decays rapidly and was not observed at $x / D=2.5$. The $u$ 'profiles were very similar to the TKE profiles (figure not shown). A comparison of the TKE and $u$ 'data showed that only $30-40 \%$ of the TKE was contained in the streamwise component over the measured region of the wake. Although not included here, the Reynolds stress invariant map in the intermediate wake region illustrated that the turbulence in the near wake region is strongly anisotropic and was primarily two-component turbulence, dominated by $u^{\prime}$ and $w^{\prime}$.

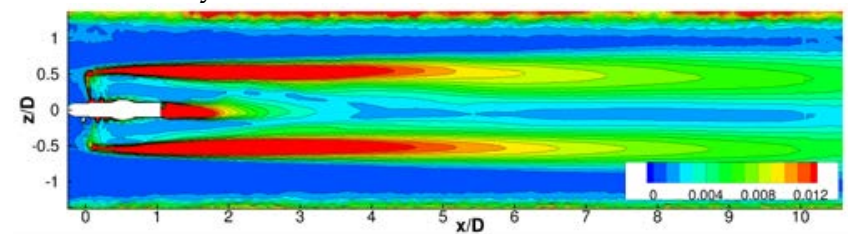

(a)

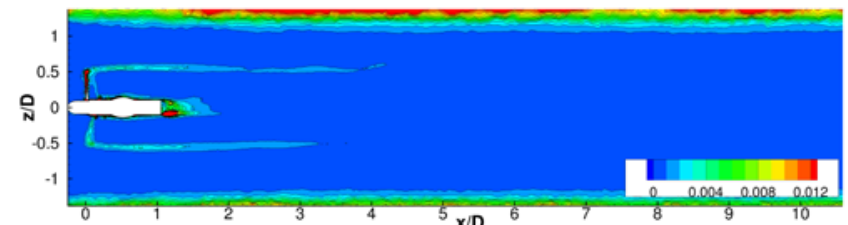

(b)

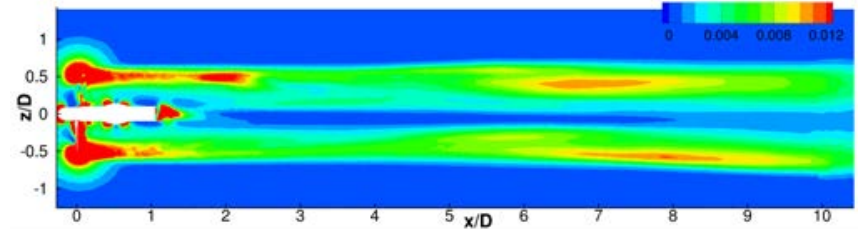

(c)

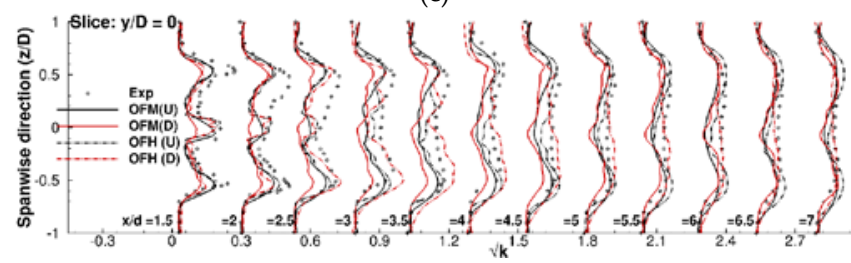

(d)

Figure 11: TKE predictions on mixed and hex cell grid are compared with experimental data. (a) Modeled TKE predicted for URANS. (b)

Modeled TKE and (c) resolved TKE in DES. (d) TKE profiles at several downstream locations are compared with experimental data.

URANS predictions showed high TKE emerging from the blade tips. The TKE levels increased further downstream and diffuses towards the center. DES also predicted high TKE in the blade tip wake. The resolved TKE was dominant in the near wake region (up to 1D), but beyond that resolved TKE was dominant and accounts for $90 \%$ of TKE. Both URANS and DES predictions compared well with experiment, except the predictions towards the center for $x / D=2.5$ to 5 .

\section{E. Effect of Free-surface on Wake}

Preliminary two-phase simulations with a tip clearance of $0.35 \mathrm{D}$ were performed to investigate the free surface and wake interactions. The free-surface wave elevation in Fig. 7(d) showed a dead water region behind the shanchion, and tip votices started to interact with the freesurface around $x / D=4$. The streamwise velocity contours for single and two-phase flow at different locations downstream are compared in Fig. 12. The results showed a strong interaction between the wake defict region and 
the free surface. The interaction between the wake and free-surface was initiated by the interaction of the stanchion with the free-surface. A significant wake deformation (skewness) was observed at $x / D=7$ which affected the wake expansion as depicted in Fig. 12(b).

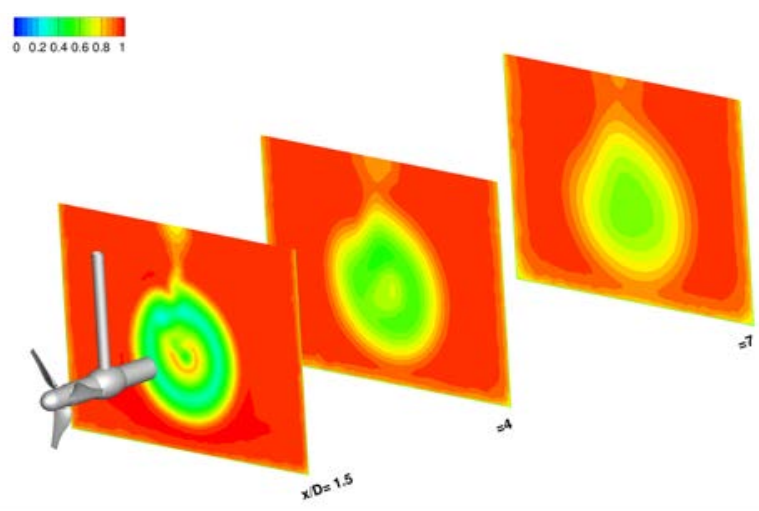

(a)

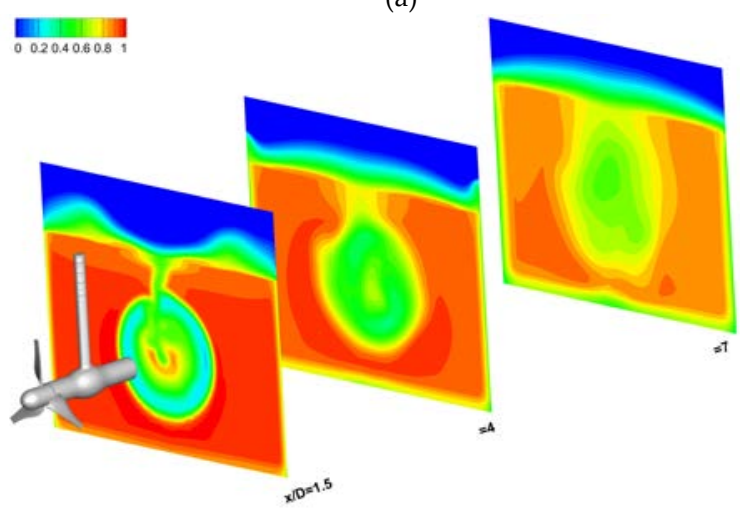

(b)

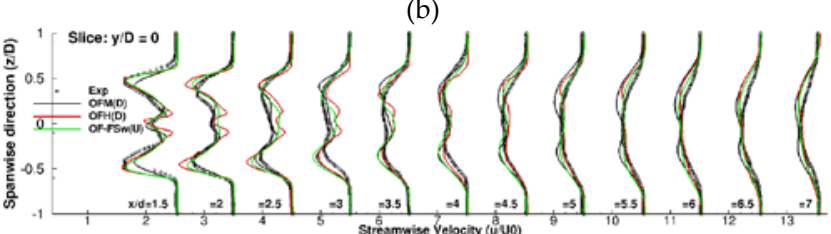

(c)

Figure 12: Wake predicted in (a) single-phase and (b) two-phase

URANS simulations are compared at $x / D=1.5,4,7$. (c) Velocity deficit profiles at several downstream locations predicted using DES on mixed $(\mathrm{M})$ and hex cell $(\mathrm{H})$ grids, and URANS free-surface (FSw) simulation are compared with experimental data. The experimental profiles were extracted from Tecplot contours files provided by the

Professor Robert Poole, and do not correspond to the actual measurement locations.

As shown in Fig. 13, the presence of the free surface creates a blockage effect which accelerates the flow in the upper bypass region. Note that the blockage occurs because the free-surface does not deform significantly. Intuitively, it is expected that the free-surface should increase downstream of the turbine due to momentum reduction, similar to the hydraulic jump phenomena. The absence of the hydraulic jump could be due to upstream flow conditions and tip-clearance gap, which needs to be further investigated. As shown in Fig. 12(c), the velocity deficit profiles obtained using the two-phase simulations compare better with the experiments than those obtained using single-phase simulations. This suggests that flow acceleration due to free-surface blockage enhances wake recovery.

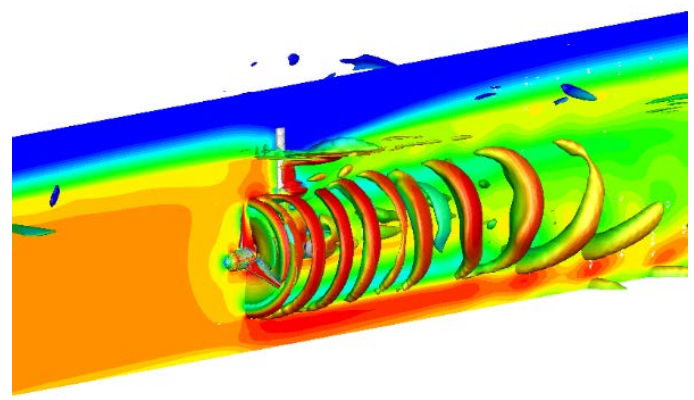

(a)

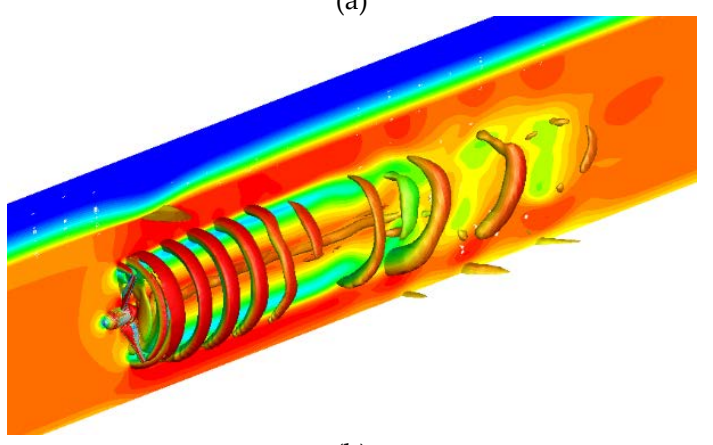

(b)

Figure 13: Velocity contours and $Q=5$ isosurfaces predicted for twophase flow: (a) with stanchion and (b) without stanchion.

\section{CONCLUSIONS}

The capability of rotating, blade-resolved simulations that employ a sliding mesh to predict HKT thrust, power and the intermediate wake was validated. For this purpose, single- and two-phase URANS and DES computations were performed for a three-blade $0.5 \mathrm{~m}$-diameter HKT turbine with a tip-speed ratio $\lambda=6.15$, and blade tip clearance of $0.35 \mathrm{D}$. The validation study focused on the prediction of turbine performance characteristics and mean and turbulent wake profiles in the intermediate wake between $x / D=1.5$ to 7 using experimental data of Tedds et al. [2].

The thrust predictions were dominated by the pressure distribution on the blades, whereas blade shear stress played a significant role in the power predictions. The mean predictions did not show a significant dependence on the turbulence modelling, and results agreed within $4 \%$ of the experimental data. However, the choice of turbulence model could be important for the operation at off-design conditions where flow separation might be present on the blades. The predictions for configurations with a stanchion showed unsteady thrust and power with unsteady amplitudes around $3 \%$ of the mean, due to the disruption of the flow each time a blade passes in front of the stanchion.

It was identified that the wake recovery was primarily due to the growth of shear layers (originating from the blade tips) towards the turbine axis, which were primarily caused by the cross-plane turbulent velocity. The shear layer growth was enhanced by the turbulence induced by the stanchion. The far-wake develops around $8 D$, showed a self-similar Gaussian deficit profile, and isotropic 
turbulence structures. OpenFOAM predictions of the mean wake profile compared within $10 \%$ of the experimental data, which was significant improvement over Fluent prediction that showed large errors of $22 \%$. The improved predictions in OpenFOAM were due to better turbulence predictions, both for URANS and DES. Preliminary two-phase results showed that the interaction of the free-surface and the wake was initiated by the stanchion, and resulted in a skewed wake. The blockage generated by the free surface accelerates the flow in the upper bypass region and enhances the wake recovery in the near and intermediate wake.

\section{ACKNOWLEDGEMENT}

All simulations were performed on Talon HPC system at High Performance computing Collaboratory, Mississippi State University. The contribution of Professor Robert Poole, School of Engineering at the University of Liverpool, who provided detailed experimental data, is gratefully acknowledged.

\section{REFERENCES}

[1] A. Kumar, et al., "Hydropower. In IPCC Special Report on Renewable Energy Sources and Climate Change Mitigation" [O. Edenhofer, R. Pichs-Madruga, Y. Sokona, K. Seyboth, P. Matschoss, S. Kadner, T. Zwickel, P. Eickemeier, G. Hansen, S. Schlömer, C. von Stechow (eds)], Cambridge University Press, Cambridge, United Kingdom and New York, NY, USA, 2011. [Online]. Available: https://www.ipcc.ch/report/renewableenergy-sources-and-climate-change-mitigation/

[2] S.C. Tedds, I. Owen, and R.J. Poole, "Near-wake characteristics of a model horizontal axis tidal stream turbine," Renewable Energy, vol. 63, pp. 222-235, 2014.

[3] D.A. Egarr, T. O'Doherty, T. Morris, and R.G. Ayre, “Feasibility study using computational fluid dynamics for the use of a turbine for extracting energy from the tide," In: 15th Australasian fluid mechanics conference. Sydney, Australia, The University of Sydney, 2004.

[4] I. Afgan, J. McNaughton, S. Rolfo, D. Apsley, T. Stallard, and P. Stansby, "Turbulent flow and loading on a tidal stream turbine by LES and RANS," International Journal of Heat and Fluid Flow, vol. 43, pp. 96-108, 2013.

[5] H.D. Nedjari, O. Guerri, and M. Saighi, "CFD wind turbines wake assessment in complex topography," Energy Convers. Manag., vol. 138, pp. 224-236, 2017.

[6] I. Bouras, L. Ma, D. Ingham, and M. Pourkashanian, “An improved $\mathrm{k}-\omega$ turbulence model for the simulations of the wind turbine wakes in a neutral atmospheric boundary layer flow," Journal of Wind Engineering \& Industrial Aerodynamics, vol. 179, pp. 358-368, 2018.

[7] Y. Liu, Q. Xiao, A. Incecik, C. Peyrard, and D. Wan, "Establishing a fully coupled CFD analysis tool for floating offshore wind turbines," Renewable Energy, vol. 112, pp. 280-301, 2017.

[8] H. Ren, X. Zhang, S. Kang, and S. Liang, "Actuator Disc Approach of Wind Turbine Wake Simulation Considering Balance of Turbulence Kinetic Energy," Energies, vol. 12, no. 16, 2019.

[9] M. Calaf, C. Meneveau, and J. Meyers, "Large eddy simulation study of fully developed wind-turbine array boundary layers," Physics of Fluids, vol. 22, no. 1, pp. 015110, 2010.
[10] C. Wang, J. Wang, F. Campagnolo, D. B. Carre'on and C. L. Bottasso, "Validation of large-eddy simulation of scaled waked wind turbines in different yaw misalignment conditions," IOP Conf. Series: Journal of Physics: Conf. Series 1037, 2018, 062007.

[11] M.J. Churchfield, Y. Li, and P.J. Moriarty, A large-eddy simulation study of wake propagation and power production in an array of tidal-current turbines, Philosophical Transactions of the Royal Society A, vol. 371: 20120421, 2013.

[12] U. Ahmed, D.D. Apsley, I. Afgan, T. Stallard, and P.K. Stansby, 3Fluctuating loads on a tidal turbine due to velocity shear and turbulence: comparison of CFD with field data," Renew. Energy, vol. 112, pp. 235-246, 2017.

[13] Y.Zhang and B. Kim, "A Fully Coupled Computational Fluid Dynamics Method for Analysis of Semi-Submersible Floating Offshore Wind Turbines Under Wind-Wave Excitation Conditions Based on OC5 Data," Appl. Sci. vol. 8, 2314, 2018

[14] A. Kasmi, and C. Masson, "An Extended k- $\varepsilon$ Model For Turbulent Flow Through Horizontal Axis Wind Turbines," Journal of Wind Engineering \& Industrial Aerodynamics, vol. 96, pp. 103-122, 2008.

[15] O. H. Espinosa, "Turbulence Modelling in Wind Turbine Wakes," Ph.D. Thesis, École de Technologie Supérieure, Montréal, QC, Canada, 2017.

[16] C. L. Archer, S. Mirzaeisefat, and S. Lee, "Quantifying the sensitivity of wind farm performance to array layout options using large-eddy simulation," Geophysical Research Letters, vol. 40, pp. 4963-4970, 2013.

[17] J.S. Moon, L. Manuel, M.J. Churchfield, S. Lee, and P.S. Veers, "Toward Development of a Stochastic Wake Model: Validation Using LES and Turbine Loads," Energies, vol. 11, no. 53, 2018.

[18] A.S. Bahaj, L.E. Myers, R.I. Rawlinson-Smith, and M. Thomson, "The effect of boundary proximity upon the wake structure of horizontal axis marine current turbines," J Offshore Mech Artic Eng, vol. 134, 2011.

[19] J. Riglin, W.C. Schleicher, I.H. Liu, and A. Oztekin, "Characterization of a micro-hydrokinetic turbine in close proximity to the free surface," Ocean Engineering, vol. 110, pp. 270-280, 2015.

[20] N. Kolekar, and A. Banerjee, "Performance characterization and placement of a marine hydrokinetic turbine in a tidal channel under boundary proximity and blockage effects," Applied Energy, vol. 148, pp. 121-133, 2015.

[21] C.L. Archera, A. Vasel-Be-Hagha, C. Yana, S. Wua, , Y. Yang Pana, J.F. Brodiea, and A.E Maguire, "Review and evaluation of wake loss models for wind energy applications," Applied Energy, vol. 226, pp. 1187-1207, 2018.

[22] S. Salunkhe, "Model- and full-scale predictions of hydrokinetic turbulent wake, including model-scale validation," MS thesis, Mississippi State University, US. 2016.

[23] H. Jasak, A. Jemcov, and Z. Tukovic "Openfoam: A C++ library for complex physics simulations," International Workshop on Coupled Methods in Numerical Dynamics, IUC, Dubrovnik, Croatia, 2007.

[24] E. Roberston, V. Choudhury, S. Bhushan, and D.K. Walters, "Validation of OpenFOAM Numerical Methods and Turbulence Models for Incompressible Bluff Body Flows," Computers and Fluids., vol. 123, pp. 122-145, 2015.

[25] A. Mason-Jones, D.M. O'Doherty, C.E. Morris, T. O'Doherty, C.B. Byrne, P.W. Prickett, et al. "Non-dimensional scaling of tidal stream turbines," Energy, vol. 44, no. 1, pp. 820-829, 2012.

[26] C. Morris, "Influence of Solidity on the Performance, Swirl Characteristics, Wake Recovery and Blade Deflection of a 
Horizontal Axis Tidal Turbine," PhD thesis. Cardiff University, UK. 2014.

[27] M. Bastankhan and F. Porte-Agel "A new analytical model for wind turbine wakes," Renewable Energy, vol. 70, pp. 116-123, 2014.

[28] Pointwise, Inc. (2007). Pointwise [Computer software]. Fort Worth, Texas, USA. https://www.pointwise.com/
[29] S. Salunkhe, O. El Fajri, S. Bhushan, D. Thompson, T. O'Doherty, D. O'Doherty, and A. Mason-Jones, “Validation of hydrokinetic turbulent wake predictions and analysis of wake recovery mechanism," Journal of Marine Science and Engineering, vol. 7, no. 10, 362, 2019.

[30] S. Bhushan, P. Carrica, J. Yang, and F. Stern, "Scalability studies and large grid computations for surface combatant using CFDShip-Iowa," The International Journal of High-Performance Computing Applications, vol. 25, no. 4, pp. 466-487, 2011. 